\title{
Utilização das Ferramentas do MASP para Aumento de Produtividade de Máquina de Corte e
} Dobra

Use of MASP Tools to Increase Productivity of Cutting and Bending Machine

Ramon Souza Lobo ${ }^{1}$, Centro Universitario Farias Brito, Dep. de Engenharia de Produção

Mauricio Johnny Loos², Centro Universitario Farias Brito, Dep. de Engenharia de Produção

\section{RESUMO}

Devido à grande concorrência no mercado do aço brasileiro, principalmente pela entrada de produtos importados da China e da Turquia, e a recessão da economia brasileira que atinge diretamente o mercado da construção civil, o qual se vê obrigado a buscar produtos com custos cada vez menores, empresas são impulsionadas a buscar um aumento de produtividade para tentarem ser competitivas. A produtividade da indústria brasileira não demonstra poder de reação ao longo dos anos, o que torna alguns negócios inviáveis frente ao aumento de custo/inflação. Neste artigo busca-se resolver o problema da baixa produtividade em uma máquina de Corte e Dobra, utilizando para isso o método do MASP e as ferramentas da Qualidade. Faz-se uma análise das principais causas para esta improdutividade utilizando-se o Diagrama de Pareto e em seguida são analisados os problemas relacionados à essas causas com a ajuda do Ishikawa. São mostrados os parâmetros de produtividade antes do trabalho ser iniciado e, depois de algumas ações sugeridas serem implementadas, novas verificações são feitas. O aumento nos números de produtividade serve para comprovar que a aplicação das ferramentas gerou resultado,

Palavras-chave: Produtividade. Corte e Dobra. MASP. Ferramentas de Qualidade.

\section{ABSTRACT}

Due to the strong competition in the Brazilian steel market, the result, principally, of the entry of products imported from China and Turkey, as well as the recession in the country that directly affects the construction market, which is forced to look for products with lower and lower costs, companies are driven to seek increased productivity to try and stay competitive. The productivity of the Brazilian industry has not shown effective reactive power over the years, which makes some businesses inviable in the face of rising cost / inflation. In this article, we try to solve the problem of low productivity in a Cutting and Folding machine, using the MASP method and the Quality tools. An analysis of the main causes for this unproductivity is performed using the Pareto Diagram; then the problems related to these causes are analyzed with Ishikawa's help. The productivity parameters are shown before the work is started and, after some suggested actions are implemented, new checks are made. The increase in the productivity numbers serves to prove that the application of the tools has generated results.

Keywords: Productivity. Cut and fold. MASP. Quality Tools

1. rslobo1@hotmail.com; 2. Avenida Rosário Congro, 2827 - Bairro Jardim Angélica - Três Lagoas/MS - CEP: 79611-009. 2. mauricioloos@hotmail.com

LOBO, R. S.; LOOS, M. J. Utilização das ferramentas do MASP para aumento de produtividade de máquina de corte e dobra. GEPROS. Gestão da Produção, Operações e Sistemas, v. 14, n. 2, p. 95 - 107, 2019.

DOI: $10.15675 /$ gepros.v14i2.2187
Editor Responsável: Prof. Dr. Hermes Moretti Ribeiro da Silva 


\section{INTRODUÇÃO}

Até pouco tempo atrás os debates sobre a produtividade na economia brasileira eram restritos a um círculo pequeno de interessados, mas vê-se nas últimas décadas esse interesse aumentar substancialmente, seja no ambiente acadêmico, entre os formuladores de políticas e principalmente no meio empresarial. Sabe-se que entre as décadas de 1970 e 2000 temas como o combate à inflação e a redução da desigualdade social ocuparam lugares de destaque na sociedade, obscurecendo assim a preocupação com avanços de produtividade.

Nos anos 2000, o país passou por um ciclo de crescimento com distribuição de renda que foi fortemente baseado na expansão da demanda, tanto externa, por commodities, quanto doméstica, derivada do aumento da renda e da incorporação de mais pessoas ao mercado de trabalho e de consumo. Depois da crise de 2008, entretanto, esse processo parece ter perdido a capacidade de, isoladamente, impulsionar o crescimento da economia. Até mesmo as variáveis demográficas e do mercado de trabalho que, durante os últimos anos contribuíram para alavancar o crescimento econômico, chegaram aparentemente ao seu limite ou próximo dele. Além disso, o investimento também não chegou a crescer acima do que tem sido seu patamar histórico nas últimas décadas, patamar este que é - e isso é praticamente um consenso entre os economistas - insuficiente para sustentar o crescimento da economia no longo prazo. É nesse cenário que a produtividade volta a ganhar relevância no debate econômico brasileiro. Não porque seu desempenho no período recente seja diferente do observado nas últimas décadas, mas porque o crescimento da produtividade se impõe novamente como uma condição para o crescimento da economia. De fato, não há nada de novo em relação a essa variável: o seu desempenho nos últimos anos não é particularmente pior, ou melhor, do que tem sido há décadas.

Já que a ampliação da produtividade será crucial para o país crescer de forma sustentada no futuro, e por muitas ocasiões será fundamental para que empresas se mantenham no mercado, este artigo tem como objetivo usar o método do MASP e ferramentas de qualidade para alavancar a produtividade de uma determinada área de uma indústria de aço.

Para cumprir seus objetivos, o trabalho primeiramente estabelece a revisão da literatura, seguida pelos procedimentos metodológicos adotados, resultados empíricos e, finalmente, suas conclusões.

GEPROS. Gestão da Produção, Operações e Sistemas, v. 14, nº 2, p. 95 - 107, 2019. 


\section{REFERENCIAL TEÓRICO}

A seguir é apresentada uma fundamentação teórica referente ao MASP, no intuito de estabelecer a visão da literatura para, posteriormente, poder-se efetuar as análises de confronto com o trabalho de campo observado no objeto de análise.

\subsection{MASP (Metodologia de Análise e Solução de Problemas)}

O MASP é um caminho ordenado, composto de etapas pré-definidas para a escolha de um problema, análise de suas causas, determinação e planejamento de um conjunto de ações que consistem uma solução, verificação do resultado da solução e realimentação do processo para a melhoria do aprendizado e da própria forma de aplicação em ciclos posteriores. O MASP prescreve como um problema deve ser resolvido e não como ele é resolvido, contrapondo dois modos de tomada de decisão que Bazerman (2004) denomina de "modelo prescritivo" e "modelo descritivo". O MASP segue o primeiro modelo (prescritivo) e, por esse motivo, é também definido como um modelo racional. Partindo também do pressuposto de que para toda solução há um custo associado, a solução que se pretende descobrir é aquela que maximize os resultados, minimizando os custos envolvidos. Há, portanto, um ponto ideal para a solução, em que se pode obter o maior benefício para o menor esforço, o que pode ser definido como decisão ótima.

De acordo com Oribe (2013), o MASP é um método prescritivo, racional, estruturado e sistemático para o desenvolvimento de um processo de melhoria num ambiente organizacional, visando solução de problemas e obtenção de resultados otimizados. O MASP se aplica aos problemas classificados como "estruturados", cujas causas sejam comuns e soluções sejam desconhecidas, que envolvam reparação, melhoria ou performance e que aconteçam de forma crônica. Pode-se perceber que para serem caracterizados da forma acima, os problemas precisam necessariamente possuir um comportamento histórico. Devido a esse fato, o MASP se vale de uma abordagem que se caracteriza como "reativa", o que contrasta com a abordagem "proativa" necessária aos problemas de engenharia ou de concepção.

O MASP é uma metodologia que auxilia os gestores na avaliação do processo e identificação das causas, objetivando encontrar soluções que possam reduzir ou eliminar tais problemas (MORAES; BORGES; SÁ, 2010). O MASP é uma metodologia de melhoria contínua, que parte da ideia de que qualquer atividade e resultado sempre podem ser GEPROS. Gestão da Produção, Operações e Sistemas, v. 14, nº 2, p. 95 - 107, 2019. 
melhorados. Para isso é necessário que a atividade de melhoria seja sistematicamente planejada (AGUIAR, 2004).

Segundo Campos (2004), a metodologia MASP possui oito etapas para a sua aplicação, onde Na Figura 1 é possível identificar as etapas e a descrição de cada uma delas dentro do processo de análise para a busca de soluções dos problemas em qualquer segmento de trabalho, na fabricação de produtos ou na prestação de serviços.

Figura 1 - Fases do MASP, objetivos e correlação com o ciclo PDCA.

\begin{tabular}{|c|c|c|}
\hline Ciclo & Fases do MASP & Objetivo \\
\hline \multirow{4}{*}{$\mathbf{P}$} & Identificaşão do problema & $\begin{array}{l}\text { Definir claramente o problema e reconhecer sua } \\
\text { importância }\end{array}$ \\
\hline & Observação & $\begin{array}{l}\text { Investigar as caracteristicas especificas do problema } \\
\text { com uma visão ampla e sob vários pontos de vista }\end{array}$ \\
\hline & Análise & Descobrir as causas fundamentais \\
\hline & Plano de açăo & $\begin{array}{l}\text { Conceber um plano para bloquear as causas } \\
\text { fundamentais }\end{array}$ \\
\hline $\mathbf{D}$ & Ação & Bloquear as causas fundamentais \\
\hline $\mathbf{C}$ & Verificaşăo & Verificar se o bloqueio foi efetivo \\
\hline \multirow[b]{2}{*}{ A } & Padronização & Prevenir contra o reaparecimento do problema \\
\hline & Conclusăo & $\begin{array}{l}\text { Recapitular todo o processo de soluçăo do } \\
\text { problema para trabalho futuro }\end{array}$ \\
\hline
\end{tabular}

Fonte: Campos (2004, p.67).

Os autores França Versiani, Yoschihiro Oribe e Loureiro Rezende (2013) explicam cada uma destas oito etapas citadas por Campos (2004):

Etapa 1 - Identificação do problema: consiste na definição clara do problema e da sua importância. Nessa etapa deve-se definir claramente o seu problema (não conformidade) para enfrentá-lo nas etapas posteriores;

Etapa 2 - Observação do fenômeno: o problema deve ser observado de forma sistêmica, coletando-se todas as informações relevantes para a sua solução. Momento de se realizar uma observação profunda sobre o problema em questão para que a etapa posterior de análise possa ser efetuada com o máximo de informações possíveis;

Etapa 3 - Análise do Processo: consiste em descobrir as causas fundamentais do problema. Na etapa de análise, o objetivo é descobrir as suas possíveis causas fundamentais. Tratando estas causas, será possível solucionar o problema de forma eficaz;

Etapa 4 - Plano de Ação: desenvolver um plano, ou procedimento que possibilite a solução do problema. Nesta etapa elabora-se o plano de ação (pode ser mais de um) para se abordar as causas fundamentais do problema, definir responsabilidades, estabelecer prazos, levantar custos, eleger métodos de execução e indicadores para monitorar a eficácia da ação; GEPROS. Gestão da Produção, Operações e Sistemas, v. 14, nº 2, p. 95 - 107, 2019. 
Etapa 5 - Ação: nesta etapa coloca-se em execução o plano de ação, para bloquear as causas raízes do problema;

Etapa 6 - Verificação: o plano de ação resolveu o problema? Realiza-se aqui o controle das ações, verificando se os planos de ação e cronogramas foram executados e se seus resultados foram satisfatórios em atender as demandas iniciais;

Etapa 7 - Padronização: adotar como padrão o procedimento elaborado no plano de ação. Uma vez que a solução do problema foi alcançada é importante padronizar o seu sucesso. O objetivo é evitar que o problema volte a ocorrer. É o momento de mapear, documentar e implementar esse processo;

Etapa 8 - Conclusão: avaliar a aplicação do método para este problema e, fortalecendo as lições aprendidas. Esta é a etapa apropriada para a gestão do conhecimento. Deve-se documentar e refletir sobre os problemas, utilizando essa reflexão como insumo para o planejamento de futuros projetos e processos.

Ao aplicar o MASP para resolver problemas o usuário precisa inevitavelmente utilizar algumas ferramentas da qualidade. Isso significa que apenas o emprego do método não é suficiente para resolver problemas de forma efetiva. É preciso que as ferramentas auxiliem o processo, fazendo algo que o método não é capaz.

A primeira coisa a ser esclarecida é a diferença entre método e ferramenta. O método é o caminho lógico, estruturado na forma de uma sequência de etapas previamente definidas e que seria o melhor roteiro para se chegar ao resultado esperado. O método é como um mapa que indica o melhor trajeto e que sempre será utilizado, pois ele foi estudado e escolhido dentre algumas alternativas possíveis. Já as ferramentas são instrumentos de trabalho de diversos tipos e que são empregados diversas vezes durante o trajeto.

Os métodos e técnicas, também conhecidos como ferramentas da qualidade, no controle de desperdícios ou até mesmo a instituição do uso de indicadores, ajudam a organização a perceber a eficácia dos processos (HIDALGO MARTINS et al., 2013). Existe uma infinidade de ferramentas, e Nancy Tague enumera quase 100 delas em seu livro "The Quality Tool Box", sem contar as variantes, que são ferramentas derivadas dos originais com pequenas alterações feitas para um propósito específico. Na Figura 2 são apresentadas sete dessas ferramentas.

Figura 2 - Ferramentas de qualidade. 


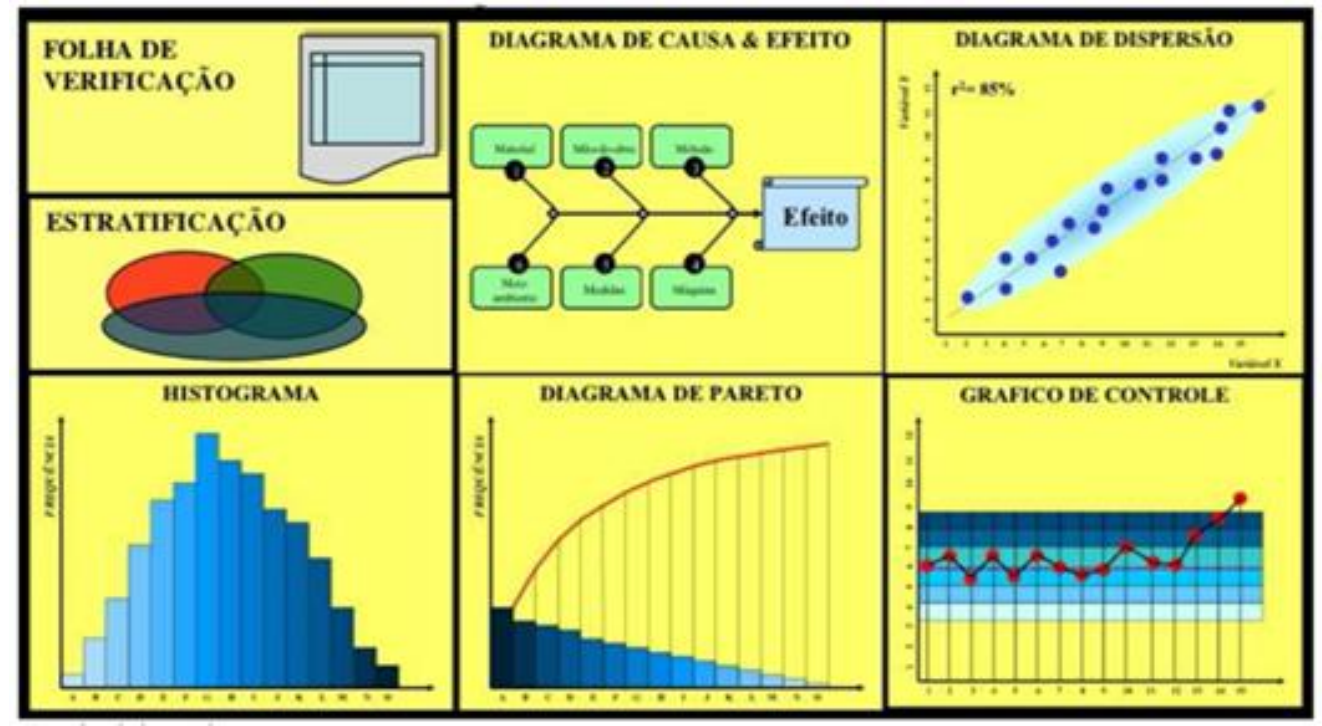

Fonte: Nancy Tangue (2005).

Segundo Werkema (1995), essas sete ferramentas da qualidade podem ser descritas da seguinte forma:

a) Folha de verificação: seu principal objetivo é facilitar da melhor forma possível a coleta de dados e organizar esses dados durante a coleta, eliminando a necessidade de retrabalhos manuais. Esta ferramenta funciona como um formulário com os itens a serem investigados já impressos, de forma a facilitar a coleta de dados;

b) Diagrama de causa e efeito: conhecido também como Diagrama de Ishikawa, esta ferramenta é usada para pontuar e apresentar as possíveis causas para um determinado problema e assim poder listar as medidas corretivas que deverão ser adotadas;

c) Diagrama de dispersão: é usada quando se tem a necessidade de visualizar o que acontece com uma variável quando uma outra variável sofre algum tipo de alteração. Dessa forma é possível saber se as duas possuem algum tipo de correlação (uma característica da qualidade e um fator que a afeta);

d) Estratificação: consiste na divisão de um grupo em diversos subgrupos com base em fatores apropriados, conhecidos como fatores de estratificação, como: métodos, medidas, equipamentos, insumos, pessoas e condições ambientais;

e) Histograma: seu objetivo é conhecer as características de distribuição associada a alguma população de interesse, neste caso se retira uma amostra desta população e se mede, para os elementos da amostra, os valores assumidos pela variável considerada; 
f) Diagrama de Pareto: consiste em um gráfico de barras verticais informando através de números percentuais de forma a evidenciar os maiores índices de causas e assim priorizálos. O princípio de Pareto estabelece uma relação 80/20, ou seja, para a resolução de $80 \%$ dos problemas deve-se atacar $20 \%$ das causas das perdas que a empresa vem sofrendo com a ocorrência dos problemas indicados;

g) Gráfico de controle: também conhecido como carta de controle, essa ferramenta consiste em identificar a variabilidade do processo e assim poder-se traçar alternativas para a estabilização do mesmo.

\section{PROCEDIMENTOS METODOLÓGICOS}

O MASP é um caminho ordenado, composto de passos e sub-passos pré-definidos para a escolha de um problema, análise de suas causas, determinação e planejamento de um conjunto de ações que consistem uma solução, verificação do resultado da solução e realimentação do processo para a melhoria do aprendizado e da própria forma de aplicação em ciclos posteriores (ORIBE, 2013).

Nesse contexto, será utilizado o estudo de caso como metodologia neste artigo, observando que o mesmo se trata de uma abordagem metodológica de investigação especialmente adequada quando procura-se compreender, explorar ou descrever acontecimentos e contextos complexos, nos quais estão simultaneamente envolvidos diversos fatores (ARAUJO, 2008).

A empresa na qual coletaram-se os dados é uma Siderúrgica multinacional, com sede no Rio Grande do Sul, presente em 14 países e com cerca de 40 mil funcionários. No estado do Ceará, iniciou sua operação em 1982 para atender a demanda dos setores de construção civil e da indústria da região por meio das mais modernas tecnologias industriais. Em 2001 entrou em operação o Centro de Serviços de Corte e Dobra de Aço, voltado para a construção civil, indústria civil e mercado eólico. Por sinal, essa é a área que será utilizada para o estudo de caso.

Para coleta de dados, foram utilizadas as informações do sistema de produção, preenchendo uma planilha criada para cálculo do indicador que será utilizado para medir a produtividade. Logo em seguida fez-se as primeiras visitas ao local afim de iniciar-se a etapa de verificação do problema. 
A partir dos dados de tempo de operador na máquina e produção, dada em toneladas, calculou-se um indicador chamado $\mathrm{kg} / \mathrm{h}$, o qual mostra quantos quilos consegue-se produzir a cada hora de produção. A Figura 3 mostra os dados coletados da estação de trabalho FSA 01 entre os meses de junho e dezembro de 2016. A produtividade média entre esses seis meses foi de $1.591,3 \mathrm{Kg} / \mathrm{h}$.

Figura 3 - Dados de produção.

\begin{tabular}{|c|c|c|c|c|c|}
\hline Mès & Estação de trabalho & Peso Produzido (T) & Tempo & $\begin{array}{c}\text { Conversão } \\
\text { em horas (h) }\end{array}$ & Produtividade Kg/h \\
\hline Junho & FSA01 & 432,58 & $298: 37: 06$ & 298,6 & 1448,6 \\
\hline Julho & FSA01 & 488,52 & $324: 15: 20$ & 324,3 & 1506,6 \\
\hline Agosto & FSA01 & 565,27 & $364: 46.12$ & 364,8 & 1549,7 \\
\hline Setembro & FSA01 & 558,40 & $335: 53: 35$ & 335,9 & 1662,5 \\
\hline Outubro & FSA01 & 379,05 & $246: 21: 06$ & 246,4 & 1538,6 \\
\hline Novembro & FSA01 & 551,17 & $299: 34: 40$ & 299,6 & 1839,9 \\
\hline Dezembro & FSA01 & 411,54 & $258: 17: 55$ & 258,3 & 1593,4 \\
\hline
\end{tabular}

Fonte: Os autores (2017).

Através das informações que são lançadas no sistema pelo próprio operador, conseguiu-se levantar todos os eventos que ocorreram no segundo semestre de 2016 na máquina FSA01. A Figura 4 apresenta o Pareto com as principais causas de interrupção de produção neste período.

Figura 4 - Pareto principais paradas.

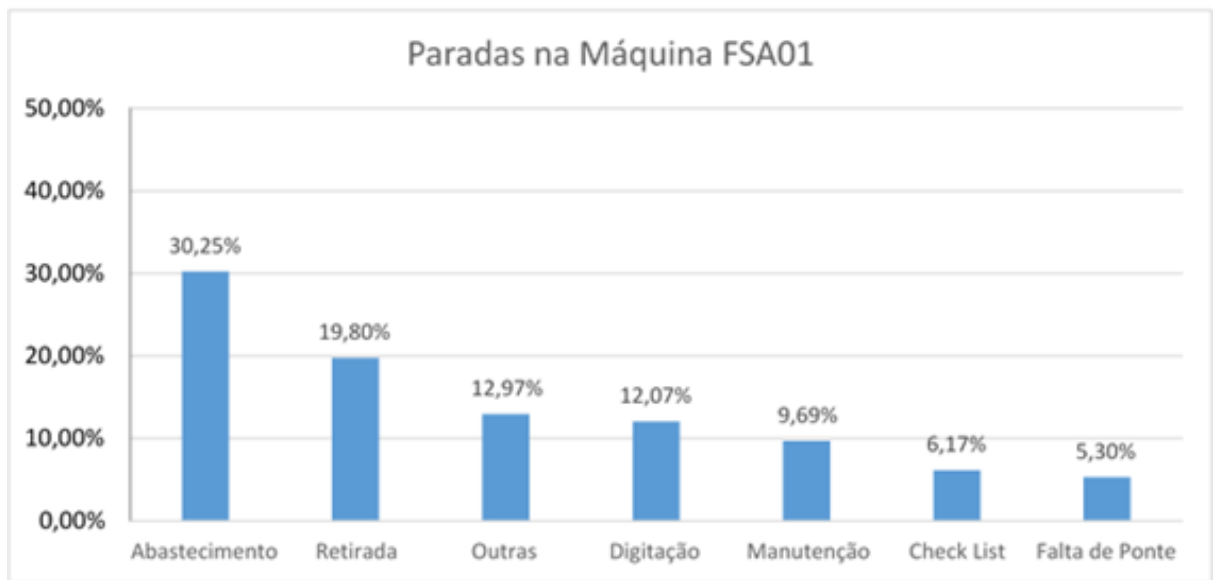

Fonte: Os autores (2017).

Os dados de parada de máquina serviram como base para definir-se em quais tarefas desenvolvidas pelo operador seria iniciada a investigação. Para isso foi aplicado o diagrama de Ishikawa, também conhecido como "espinha de peixe", onde através dessa ferramenta pode-se traçar as ações focando nos seis pontos principais que a ferramenta 
trabalha, os quais são: mão de obra, máquina, método, meio ambiente, matéria-prima e medição, os quais também são conhecidos como 6 Ms. Essa análise pode ser vista na Figura 5.

Figura 5 - Diagrama de Ishikawa.

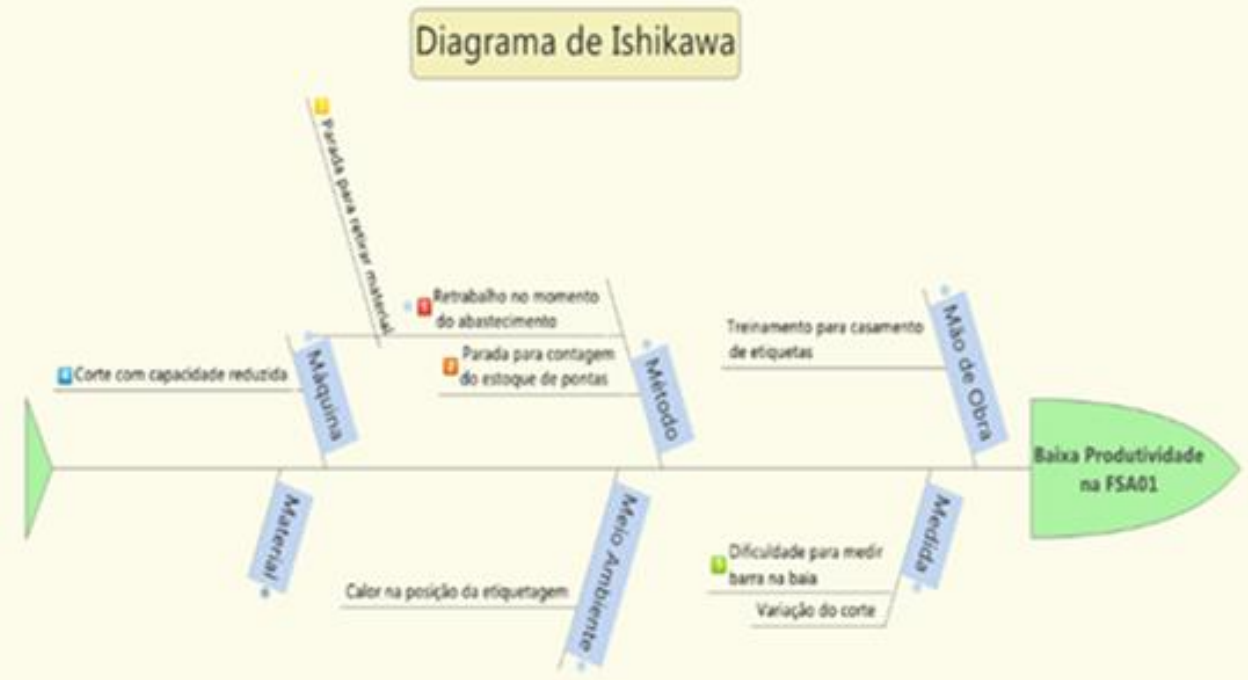

Fonte: Os autores (2017).

Na reunião de brainstorming foram levantadas algumas possibilidades para a perda de produtividade e, a partir daí, visitas à área foram planejadas afim de confirmar a relevância de cada causa para o trabalho de aumento de produtividade.

\section{RESULTADOS E DISCUSSÕES}

Foram feitas diversas visitas a campo para cumprir a etapa de observação do problema. A máquina foi visitada em horários diversos, inclusive no turno noturno, afim de serem identificadas se as práticas entre equipes eram semelhantes.

De acordo com o Pareto com as principais causas de parada e os recursos disponíveis para serem trabalhados nas soluções, foram escolhidas cinco causas que contribuem para a baixa produtividade do objeto de estudo. Essas cinco causas e as sugestões para resolução estão descritas a seguir.

\subsection{Medição}

Dentro do parâmetro de medição, durante a etapa de observação o operador necessita acessar as baias para conferir se o corte foi feito com o comprimento correto, e para isso ele utiliza uma trena de 8 metros. A trena dificulta a medição, pois além de por várias vezes ela 
"escapar", alguns materiais têm mais de 8 metros e o operador precisa realizar a tarefa em duas etapas. Sugere-se para este problema a substituição da trena comum utilizada por uma trena a laser.

\subsection{Método}

Quando se fala de métodos, foram detectados três problemas que impactam na produtividade, conforme descritos a seguir:

a) Parada da máquina para contagem do estoque de ponta: todos os dias a máquina ficava parada por 30 minutos para alguém acessar a área do estoque de pontas e efetuar a atualização do estoque. Como este estoque é secundário, de matéria prima, foi alterado o procedimento para que esta contagem fosse feita apenas uma vez por semana;

b) Retrabalho no momento do abastecimento: quando a máquina é abastecida, geralmente com dois feixes de 2 toneladas cada, o operador acessa a baia móvel apenas para retirar as cintas de içamento, deixando a etapa de corte das amarras para outro momento e parando a máquina novamente para isso. Recomenda-se que seja alterado o padrão para que os dois operadores acessem a baia móvel no momento do abastecimento, e enquanto um operador retira as cintas de içamento, o outro efetua o corte das amarras, evitando assim nova parada da máquina;

c) Parada para retirar material: quando o operador de ponte rolante chega para retirar o material da máquina, a mesma fica parada e ele mesmo passa as cintas no material para movimentação. As cintas já poderiam ficar nas baias de forma que, depois de o material ser produzido, o mesmo já caia na cinta, diminuindo assim o tempo de içamento e movimentação.

\subsection{Máquina}

$\mathrm{Na}$ etapa de identificação do problema foram buscadas as especificações da máquina e os limites de corte determinados pelo fabricante. Nesse momento, identificou-se que há muito tempo os operadores efetuavam os cortes sempre com uma barra a menos. 
Foi investigada a causa para este comportamento e concluiu-se que além do desconhecimento técnico da especificação, o equipamento apresentava problemas mecânicos que não permitiam que o sistema de arraste e corte operasse com toda a sua capacidade. As correções necessárias foram feitas para que a máquina voltasse a operar em sua capacidade máxima.

\subsection{Evolução dos resultados}

Ao final da implantação das ações sugeridas neste artigo, entrou-se na etapa de verificação. Foram acompanhados os valores de produtividade nos quatro meses seguintes e percebeu-se um aumento na ordem de $15,7 \%$, saindo de $1.591,3 \mathrm{Kg} / \mathrm{h}$ para $1.840,6 \mathrm{Kg} / \mathrm{h}$, conforme mostra a Figura 6.

Figura 6 - Produtividade mensal.

\begin{tabular}{|c|c|c|c|c|c|}
\hline Mês & Estação de trabalho & Peso Produzido (T) & Tempo & $\begin{array}{c}\text { Conversão em } \\
\text { horas (h) }\end{array}$ & Produtividade $\mathrm{Kg} / \mathrm{h}$ \\
\hline Janeiro & FSA01 & 670,85 & $328: 12: 11$ & 328,2 & 2044,0 \\
\hline Fevereiro & FSA01 & 266,20 & $162: 32: 44$ & 162,5 & 1637,9 \\
\hline Março & FSA01 & 508,87 & $278: 42: 13$ & 278,7 & 1825,9 \\
\hline Abril & FSA01 & 465,24 & $250: 51: 37$ & 250,9 & 1854,7 \\
\hline
\end{tabular}

Fonte: Os autores (2017).

$\mathrm{O}$ resultado ainda poderá ter ganhos à medida que outras ações menores e com necessidades de investimento financeiro forem sendo implementadas.

\section{CONCLUSÕES}

Por meio da utilização do método MASP é possível analisar um determinado problema de forma embasada, tendo-se condições de investigar as causas do problema e propor soluções para eliminá-las. Para isto, é imprescindível que haja a colaboração de todas as pessoas que possam contribuir com sugestões, informações, conhecimento técnico e prático, e principalmente da liderança, sendo esta a porta voz destas sugestões.

Após serem analisados os resultados obtidos, pode-se afirmar que o objetivo do trabalho foi alcançado, pois, por meio do método do MASP e das ferramentas de qualidade, a produtividade da área estudada na indústria de aço teve impactos positivos. Diversas ações sugeridas no artigo foram implantadas e algumas estão em andamento. Novos padrões foram GEPROS. Gestão da Produção, Operações e Sistemas, v. 14, nº 2, p. 95 - 107, 2019. 
Utilização das Ferramentas do MASP para Aumento de Produtividade de Máquina de Corte e Dobra

escritos e a equipe foi treinada novamente, para seja estímulo à novas aplicações em outros setores da organização.

\section{Referências}

AGUIAR, P. C. G. Aplicação da metodologia, de análise e solução de problemas na célula lateral de uma linha de produção automotiva. 2004. 65f. Monografia (Especialização em Gestão Industrial) - Departamento de Economia, Contabilidade e Administração da Universidade de Taubaté, Taubaté, São Paulo, 2004.

ARAÚJO, C. Estudo de caso. Métodos de Investigação em Educação. Portugal, 2008, p. 323 , 
CAMPOS, V. F. Controle da Qualidade Total (no estilo japonês). Belo Horizonte: Nova Lima, 2004.

CAMPOS, V. F. Gerenciamento da rotina do trabalho do dia a dia. Belo Horizonte: Desenvolvimento Gerencial, 2004.

FRANÇA VERSIANI, Â.; YOSCHIHIRO ORIBE, C.; LOUREIRO REZENDE, S. F. A aprendizagem das organizações gerada pelas práticas formais no ambiente de trabalho. RAM. Revista de Administração Mackenzie, v. 14, n. 4, 2013.

MARTINS, G. H. H.; LIMA, T. S.; TIGRINHO, C. E.; KOSSOWISKI, L. Projeto de redução do desperdício de matéria-prima: estudo de caso na indústria de embalagens de papel no Brasil. Revista de Gestão e Projetos, v. 4, n. 3, p. 141, 2013.

MORAES, M. A. G.; BORGES, E. C. B.; SÁ, J. A. S. Aplicação da Metodologia Masp para redução das perdas na produção de cabos de ferramentas agrícolas: um estudo de caso. In: ENCONTRO NACIONAL DE ENGENHARIA DE PRODUÇÃO. 30. 2010. Anais...ENEGEP, São Carlos, SP, Brasil.

ORIBE, C. Descrição e Análise Detalhada do MASP, 2013. Disponível em: http://www.qualypro.com.br/adminqualypro/upload/arquivo?nome=71.pdf\&dir=pdf. Acesso em $25 / 08 / 17$.

WERKEMA, M.C.C. As Ferramentas da Qualidade no Gerenciamento de Processos, $1995 . \quad$ Disponível em: http://www.cesumar.br/prppge/pesquisa/epcc2011/anais/sheila_luz2.pdf. Acesso em 25/08/17. 